\title{
Overexpression of Her1 (EGFR) in Gastric Cancer: A Saudi Regional Population Based Study ${ }^{*}$
}

\author{
Ayesha Ahmed $^{1 \#}$, Dalal M. Al Tamimi ${ }^{1,2}$ \\ ${ }^{1}$ Anatomic Pathology, University of Dammam, Dammam, KSA; ${ }^{2}$ King Fahd Hospital of the University, Khobar, KSA. \\ Email: \#ayesash@hotmail.com, dalaltam@yahoo.com
}

Received June 26 ${ }^{\text {th }}$, 2012; revised July 30 ${ }^{\text {th }}$ 2012; accepted August $14^{\text {th }}, 2012$

\begin{abstract}
Background: Gastric cancer is one of the commonest malignant tumor worldwide. Its treatment remains a challenge for physicians. Epidermal growth factor receptor (EGFR) inhibitors have played a significant role in the management of solid malignancies including colorectal cancer. In this study we aimed to determine EGFR expression in gastric adenocarcinoma by standardized immunohistochemistry in a Saudi regional population based cohort and also to evaluate Ki-67 proliferating index and p-53 mutation status. Materials and Methods: Gastric carcinoma (GC) cases comprising surgical resection specimens and endoscopic biopsies, were selected, from the pathology archives of King Fahd Hospital of the University of Dammam (KFHU), spanning a time period of 6 years. The histological GC type was delineated according to Laurens classification and immunohistochemical (IHC) protein analysis for EGFR, Ki-67 and p-53 was carried out. Results: 42 cases of gastric GC were analyzed and EGFR overexpression was demonstrated in $4.76 \%$ of cases. Out of these $2.38 \%$ had membranous and the remaining demonstrated predominantly cytoplasmic along with focal membranous positivity. Ki-67 proliferation index ranged from moderate to high and and p-53 mutation status was negative in these cases. Conclusion: Low EGFR expressivity could be reflective of regional variation in cancer characteristics. The study also highlights the inadequacy of the currently employed gastric EGFR interpretation criterions and stresses on development of standardized and uniform EGFR evaluation protocols tailored for gastric needs.
\end{abstract}

Keywords: Gastric Cancer; EGFR Overexpression; Immunocytochemistry; Protein Analysis

\section{Introduction}

Gastric cancer is one of the commonest malignant tumor worldwide and [1-3]. It ranks fourth amongst the most commonly diagnosed cancer, with more than $1,300,000$ cases diagnosed yearly $[4,5]$. The estimated incidence of gastric cancer in the United States was 21,500 in 2008 [6] and a cumulative mortality of esophageal and gastric cancer being approximately 1,100,000 [5], which emphasizes the global challenge in dealing with these diseases. This cancer epitomizes the concept of geographical variation in prevalence distribution with an incidence wise changing and shifting epidemiological trends, and currently East Asia makes up for a significant proportion of new cases [7]. Japan ranks the highest with increasing incidence also seen in many developing countries in Asia, Africa and Latin America [8].

Gastric cancer treatment remains a challenge for physicians [9]. Advanced gastric cancer is associated with poor prognosis, with the resultant, post diagnostic mean survival rate being approximately 10 to 11 months [4].

\footnotetext{
*We declare that we have no financial or non-financial competing interests. ${ }^{\#}$ Corresponding author.
}

Targeted therapies based on the evaluation and analysis of the status of target genes $[10,11]$ have been the recent addition in gastric cancer treatment. Molecular therapies constitute monoclonal antibodies or small molecule inhibitors targeting either growth factors or growth factor receptor kinases. Anti-HER2 humanized monoclonal antibody Trastuzumab has been proved to improve patients prognostics in HER2-positive gastric carcinoma [12]. Epidermal growth factor receptor (EGFR) inhibitors have played a significant role in the management of solid malignancies including colorectal cancer. Currently, there are four EGFR inhibitors approved by the FDA including two small molecule tyrosine kinase inhibitors (erlotinib and gefitinib) and two monoclonal antibodies (cetuximab and panitumumab) [13-17]. In gastric carcinoma EGFR is a new field, unraveling new therapeutic options for patients. Recapitulating the pattern of drug-diagnostic Herceptin/HercepTest, patients trialed and evaluated for anti EGFR drugs need to fulfill the prerequisite of IHC evidence of EGFR positive expression. This not only augments the pathologist role in therapy selection but also stresses on their responsibility of providing accurate and reproducible EGFR analysis results [18]. Standardiza- 
tion of EGFR immunoreactivity expression criteria in gastric cancer is undergoing trials and tribulations and requires to be tailored precisely for the endogenous gastric needs as has been accomplished for Her-2 in gastric cancer.

In our study we aimed to determine EGFR expression in gastric adenocarcinoma by standardized immunohistochemistry in a Saudi regional population based cohort and simultaneous evaluation of Ki-67 proliferating index and p-53 mutation status.

\section{Materials and Methods}

\subsection{Specimen Selection and Clinicopathological Parameters}

Gastric carcinoma cases comprising surgical resection specimens and endoscopic biopsies, were selected, from the pathology archives of King Fahd Hospital of the University (KFHU), spanning a time period of 6 years, under the approved protocols of the research and ethical committee of University of Dammam. Patient consent was waived due to loss of follow up for old cases. Only gastric adenocarcinomas with availability of representative blocks and sufficient tissue material to perform the required histopatholgical procedures were selected. Gastric neoplasia besides adenocarcinoma, metastatic cancer of the stomach from another organ and metastatic tumor of gastric origin without concomitant histological material from primary tumor in the stomach were excluded from the study. The current study comprises 42 cases of gastric adenocarcinoma

\subsection{Histological Classification of Gastric Cancer}

The histological GC type was delineated according to Laurens classification, this classification divides adenocarcinomas into two types: intestinal (consisting of well formed tubules) and diffuse (diffuse tumor infiltration without well formed tubules, frequently with signet ring cells) [19].

\subsection{Immunohistochemistry}

Immunohistochemical staining using the labeled streptavidin-biotin (LSAB) method with 3,3'-diaminobenzidine (DAB) as a chromagen was performed for EGFR (HER1), p-53 and Ki-67 on $4 \mu \mathrm{m}$ thick paraffin sections cut from conventional blocks. The staining was performed concurrently in a Ventana Benchmark automated immunostainer according to the manufacturer's instructtions (Ventana Medical Systems Inc., Strasbourg). Sources and dilutions of the primary antibodies used in the study are listed in Table 1. The immunostained sections were examined under a light microscope and evaluated manually by 2 pathologists (AA and DT). Any in
Table 1. Sources and dilutions of primary antibodies used in the study.

\begin{tabular}{cccc}
\hline Antibody & Clone & Manufacturer & Dilution \\
\hline EGFR & 5B7 & Ventana & Prediluted \\
Ki-67 & MIB-1 & Dako & Prediluted \\
p-53 & DO-7 & Dako & Prediluted \\
\hline
\end{tabular}

EGFR = epidermal growth factor receptor.

terpretational discrepancies were resolved under a double-headed microscope.

\subsection{Evaluation of Immunostaining}

EGFR: Both membranous and cytoplasmic staining were considered for evaluation. The membranous positivity for EGFR (mEGFR) was evaluated in the following manner: 0 , no discernible staining or background type staining; $1+$, equivocal discontinuous membrane staining; 2+, unequivocal membrane staining with moderate intensity; and $3+$, strong and complete plasma membrane staining. More than $10 \%$ of the cells were required to meet the criteria for EGFR analysis. Scores of $2+$ and $3+$ staining levels were considered to be EGFR overexpression [9]. For positive cytoplasmic EGFR (cEGFR) staining, an intense homogenous staining of the cytoplasm was necessary. There was no cEGFR without membrane stainin [20].

Ki-67: Positive staining was defined as positive nuclear staining. Cytoplasmic staining was considered negative. The percentage of positive nuclei was expressed as a "Ki-67 labeling index" which is the percent of cells expressing Ki-67 determined by counting 1000 cells/slide. The percentage of positive cells was scored as follows: less than $10 \%=$ low proliferative activity, $10 \%-40 \%=$ moderate proliferative activity, and more than $40 \%=$ high proliferative activity [21].

P53: Positive staining was defined as positive nuclear staining. Cytoplasmic staining was considered negative. Tumors were considered focally positive when unequivocal staining was present in $10 \%-50 \%$ of tumor cells, and as diffusely positive when more than $50 \%$ of the tumor cells were positive [22].

\subsection{Statistical Analysis}

Data was entered into SPSS windows. Frequencies were calculated using descriptive statistics for categorical variables.

\section{Results}

Out of a total of 42 cases of gastric cancer retrieved, 38 specimens were biopsies and 4 were partial gastrectomies. Male to female ratio was 25:9 and median age of the pa- 
tients was 67 years (extreme 88 - 42 years). Diffuse type gastric cancer was seen in $23(54.76 \%)$ and Intestinal type in 19 (45.23\%) of cases. EGFR expression pattern is shown in Table 2 and Figure 1. Ki-67 proliferation index and p-53 mutation status is given in Tables 3 and $\mathbf{4}$ respectively. In cases showing EGFR overexpression (n $=2$ ) $\mathrm{p}-53$ mutation status was negative and Ki-67 ranged from moderate to low.

The Figure 2 shows H \& E staining in gastric carcinoma cases while Figure 3 shows the respective immunohistochemical panels.

\section{Discussion}

We demonstrated a low EGFR overexpression by protein analysis, comprising a mere $4.76 \%$ of $2+$ intensity and none with $3+$ immunoreactivity. Out of these $2.38 \%$ had membranous and the remaining demonstrated predominantly cytoplasmic along with focal membranous positivity. This low positivity and pattern of expression arouses several discussable issues. Firstly whether this expressivity could be part of the concept of ethnic varia-

Table 2. EGFR overexpression in gastric cancer $(n=42)$.

\begin{tabular}{|c|c|c|}
\hline Staining intensity & $\mathbf{N}$ & $\%$ age \\
\hline $\mathbf{0}$ & 34 & 80.95 \\
\hline $1+$ & 6 & 14.28 \\
\hline $2+$ & 2 & 4.76 \\
\hline $3+$ & 0 & 0 \\
\hline & \multicolumn{2}{|c|}{ 1+(negative) } \\
\hline & \multicolumn{2}{|c|}{ 2+(moderately positive) } \\
\hline & $3+1$ & sitive) \\
\hline
\end{tabular}

Figure 1. EGFR overexpression in gastric cancer $(n=42)$.

Table 3. Ki-67 Proliferation Index in gastric cancer $(n=42)$.

\begin{tabular}{ccc}
\hline Proliferative activity & N & \% age \\
\hline Low & 6 & 14.28 \\
Moderate & 14 & 33.33 \\
High & 22 & 52.38 \\
\hline
\end{tabular}

Table 4. p-53 mutation status in gastric cancer $(n=42)$.

\begin{tabular}{ccc}
\hline p-53 status & N & \% age \\
\hline Negative & 22 & 52.38 \\
Focal & 10 & 23.80 \\
Diffuse & 10 & 23.80 \\
\hline
\end{tabular}

tion in cancer distribution or could it be merely reflective of the marked variations in the intrinsic protein accentuating potential by various commercially available kits. Also they grey zone of moderate cytoplasmic positivity needs to be stressed on as regards whether these tumors be trialed for further genetic evaluation and selected for anti-EGFR therapy, the sensitivity and long term prognostic benefits of which are currently being debated. Both of the cases were endoscopic biopsies and 10\% of tumor showing positivity was the cut off point. Is this criterion applicable only for the gastrectomy specimens or needs to be downgraded as in Her-2neu positivity, where presence of just 5 positive cells now justifies institution of targeted therapy in small biopsies?

Regarding tumor related ethnic variation a tremendous heterogeneity is seen in terms of epidemiology, tumor histology and expression of molecular markers in gastric cancer [23]. EGFR overexpression has been documented to show marked ethnic variations. Mammano et al. detected EGFR protein expression in only $6 \%$ of the cases, and concluded in their study that EGFR protein expression is low and specific EGFR gene mutations are very rare or absent in gastric adenocarcinoma [24]. A some what similar expression pattern is also reported by Takehana et al., who, in their series of 413 gastric carcinomas, found negative EGFR protein expression in $89.6 \%$, low level in $8.2 \%$ and high level in $2.2 \%$ of cases [25], Lee et al., who found no EGFR gene mutations in 185 gastric adenocarcinomas in a series of Korean patients [26], and by Mimori et al., who found a silent mutation in exon 20 in only 5 of $39(5.1 \%)$ Japanese patients [27]. These studies are in agreement with our study that also shows a very low EGFR expressivity but in contrast with multiple studies documenting a much higher prevalence pattern.

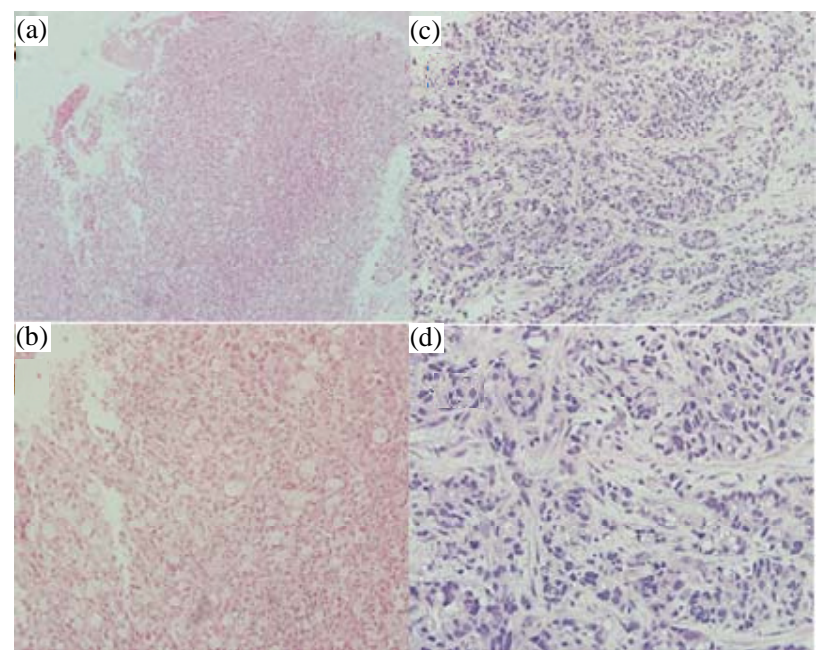

Figure 2. Gastric Carcinoma H \& E. (a) Diffuse gastric carcinoma H \& E × 20; (b) Diffuse gastric carcinoma H \& E $\times 40$; (c) Intestinal type gastric carcinoma $\mathrm{H} \& \mathrm{E} \times 20$; (d) Intestinal type gastric carcinoma $\mathrm{H} \& \mathrm{E} \times 40$. 

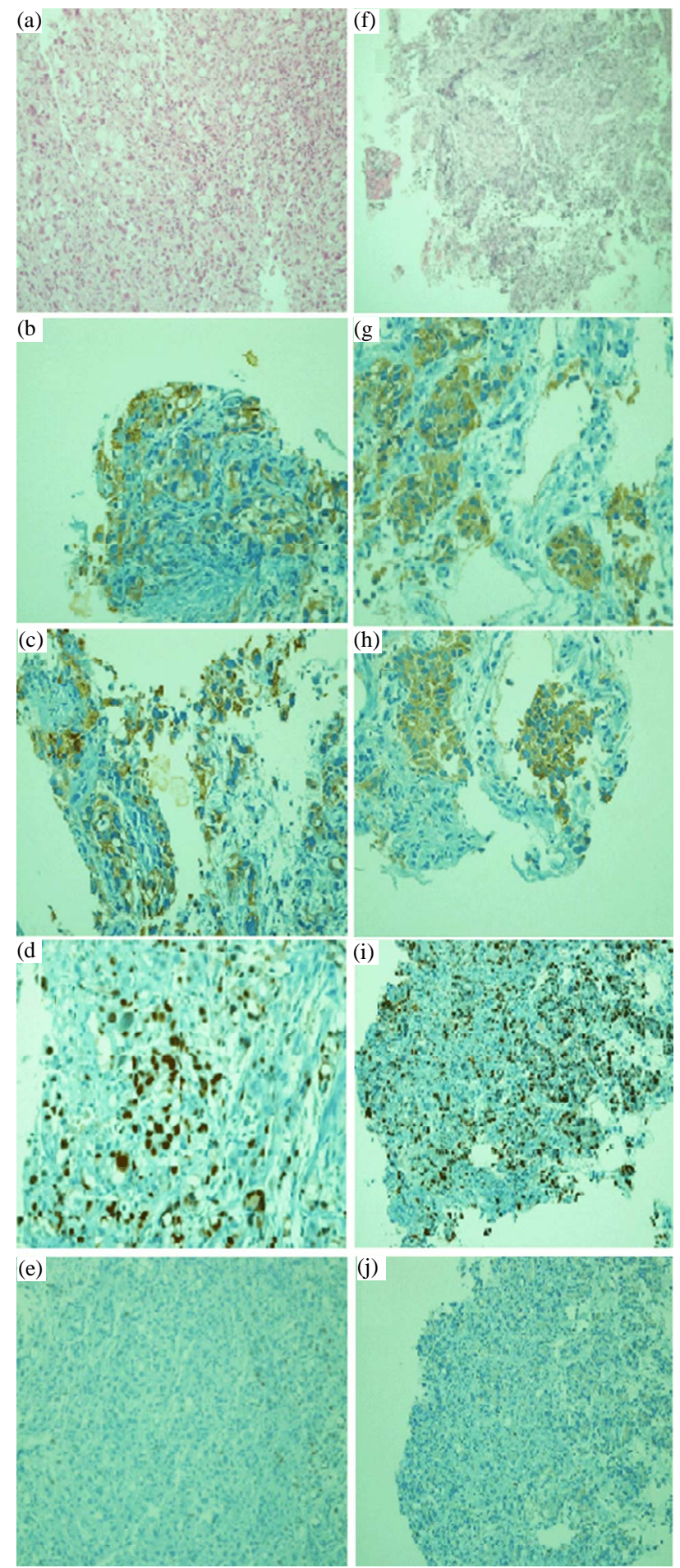

(j)

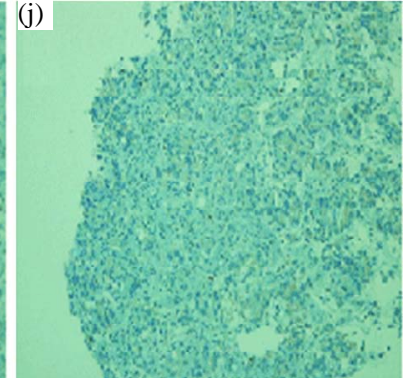

Figure 3. Gastric carcinomas with panel of IHC. (a) Diffuse gastric cancer $\times 40$ H \& E; (b) EGFR $\times 20$ 2+ (Cyto + memb); (c) EGFR $\times$ 40; (d) Ki-67 moderate; (e) p-53 negative; (f) Intestinal Type gastric cancer $\times 40$ H \& E; (g) EGFR $\times 202+$ (cyto); (h) EGFR ×40; (i) Ki-67 high; (j) p-53 negative.

Liang et al. documented $41 \%$ of EGFR expression by IHC in a subset of Chinese patients; with $16 \%$ also revealing FISH positivity [9]. In a study by Galizia et al. in an Italian population with an aim to correlate EGFR positivity by IHC with disease recurrence and survival in univariate and multivariate analyses, forty-four percent (36 cases) of gastric cancers were EGFR positive [28]. These multiple studies highlight the concept that specific EGFR mutations show a wide regional and ethnic variation in gastric adenocarcinoma, a fact substantiated in other tumors also, as demonstrated by Paez et al., who found a significant difference between the mutation rate of EGFR gene in non small cell lung carcinoma(NSCLC) from USA (2\%) and from Japan (26\%) [29].

The low EGFR expressivity pattern may also be attributed the type of kit employed for immunostaining. In a comparative study conducted by Lee $e t$ al. in NSCLC different intensities of staining were discerned by different commercially available kits. EGFR protein overexpression was observed in 56\% of tumors with Zymed EGFR kit, in 51\% with Dako EGFR pharmDx kit, in 5\% with Dako and in 18\% with Novocastra. Both Zymed and Dako pharmDx kit were more sensitive than the Dako test (clone H11) and Novocastra clone EGFR 113. They concluded that EGFR protein overexpression rate varied from $4 \%$ to $72 \%$ according to different antibody clones and histologic subtypes, and EGFR protein expression detected by Zymed and Dako pharmDx was significantly associated with a high EGFR gene copy number [30]. We had employed Ventanna kit for our study. Our pattern of expression could be attributed to the different intrinsic sensitivity of the protein highlighting potential of the kit. More extensive comparative studies regarding standardization and harmonization of the different methodologies employed for EGFR protein expression, need to be carried out so such marked variations, if existent with our logistics, may not get manifested.

Regarding membranous pattern of immunoreactivity, in our study $2.38 \%$ of cases had a heterogenous pattern of membranous staining, with mostly $2+$ pattern but with scattered individual cells showing intense complete membranous 3+ pattern of expression. These cells however did not fulfil the $10 \%$ criteria to accord $3+$ to the case as a whole. The EGFR IHC interpretation criteria in gastric cancer is essentially the same as in breast malignnancies. Does it need to be questioned, modified and downgraded as in Her-2 neu testing in gastric cancer where after validation in TOGA trial [31], the criterion is less strict and $10 \%$ cut-off rule applies to resection specimen whereas in biopsies any group of at least 5 tumour cells showing immunoreactivity satisfies the positivity standard and in addition [31] the completeness of membrane staining is not a "condition sine qua non" [12]. In EGFR IHC interpreting protocol, no such downgrdation and revision has been implemented. The intratumoral heterogeniety, more significant in small endoscopic biopsies mandates modification of expressivity 
criterions and need them to be divergent from those accepted and validated for breast cancers.

As far as cytoplasmic pattern of expression is concerned, $2.38 \%$ of cases in our study revealed, diffuse moderate cytoplasmic staining with focal membranous accentuation. Although marked, diffuse cytoplasmic staining has been considered by some studies [20] but no well defined protocols have been delineated and inclusion of these cases for further genetic evaluation and incytoplasmic positivity needs to be standardized and validated as recent studies have proved EGFR to act as a cytoplasmic/nuclear shuttling transcription factor [32] with its activation and subsequent nuclear translocalization, leading to regulation of gene expression and mediation of specific cellular processes [33,34]. This is distinct from EGFR mediated transduction of mitogenic signals through activating multiple signaling cascades [35]. Could tracking into this cytoplasmic positivity unmask a potential valid anti EGFR therapy candidate, yet needs to be ascertained. EGFR inspite of being a promising target in cancer therapy, yet failed to acquire definitive, sensitive potential accurate molecular predictors of sensitivity to EGFR inhibitors for patients having gastro-esophageal cancers [36]. The dichotomous cytoplasmic and membranous staining in other tumors holds prognostic significance as reported in renal cell carcinoma [37] with its association with worsening progression and prognosis [38]. In gastric cancer, however, such confirmation yet remains to be done.

Ki-67 proliferation index in our cases with EGFR immunoreactivity ranged from moderate to high, implicating these tumors to be aggressive. EGFR expression correlates with disease recurrence and poorer survival [28]. This is substantiated by other studies as well in which EGFR overexpression is associated with an aggressive tumor and also with lymph node metastasis [39]. EGFR dimerization and activation leads to downstream upregulation of multiple processes that can result in cancer cell proliferation, reduced apoptosis, tumor-induced angiogenesis, and activation of invasion, dedifferentiation of cancer cells and enhanced metastatic potential $[40,41]$.

The role of p-53 as a prognostic factor in gastric cancer is controversial. One school of thought describes expression of EGFR and p-53 to have a negative correlation with patients prognostics [42-44]. In a study on an arab population the expression of p53 was found to correlate with aggressive gastric cancer characteristics [45]. On the other hand, studies have reported p53 to have no influence on prognosis [46,47]. Liu et al. demonstrated p53 nuclear reactivity in association with bax and c-myc and with well-differentiated histology and with no prognostic significance [48]. In our study the overall p-53 reactivity was found to be in $47.60 \%$ of cases with diffuse positivity in $23.80 \%$ and focal positivity also in $23.52 \%$ but in both the EGFR expressing cases, it was non reactive. The cases comprised equal percentage of diffuse and intestinal types. Multiple genetic and epigenetic alterations have been found to underlie gastric carcinogenesis with different combinations becoming apparent in the two histological types of gastric cancer. p-53 mutation, reduced p27 expression, cyclin $\mathrm{E}$ expression etc in the intestinal-type and $\mathrm{LOH}$ at chromosome 17p, mutation of p53 and mutation or loss of E-cadherin to name some in the poorly differentiated gastric cancers [49]. Although p-53 mutation is seen to formulate an integral overlapping component in both the types the lack of expression in our cases could imply other molecular alterations besides p-53 mutation to be more operative.

\section{Conclusion}

Low EGFR expressivity could be reflective of regional variation in cancer characteristics. The study also highlights the inadequacy of the currently employed gastric EGFR interpretation criterions and stresses on development of standardized and uniform EGFR evaluation protocols tailored for gastric needs.

\section{Acknowledgements}

We acknowledge university of Dammam for providing financial and other logistic support for carrying out the research work. We also acknowledge services of Mrs Khalda Al Johy, Mrs Zainab Al Najar, Mr Shakir Ahmad and Mrs Maria Rosario Lazaro in conducting the laboratory work.

\section{REFERENCES}

[1] D. P. Kelsen, et al.,“Adjuvant and Neoadjuvant Therapy for Gastric Cancer,” Seminars in Oncology, Vol. 23, No. 3, 1996, pp. 379-389.

[2] D. M. Parkin, P. Pisani and J. Ferlay, "Estimates of the Worldwide Incidence of Eighteen Major Cancers in 1985,” International Journal of Cancer, Vol. 54, No. 4, 1993, pp. 594-606. doi:10.1002/ijc.2910540413

[3] D. A. Corley and P. A. Buffler, "Oesophageal and Gastric Cardia Adenocarcinomas: Analysis of Regional Variation Using the Cancer Incidence in Five Continents Database," International Journal of Epidemiology, Vol. 30, No. 6, 2001, pp. 1415-1425. doi:10.1093/ije/30.6.1415

[4] B. Hermann, R. Dietrich and A. Volker, "Epidemiology of Stomach Cancer,” Springer Protocols, Vol. 472, 2008, pp. 467-477.

[5] D. M. Parkin, F. Bray, J. Ferlay and P. Pisani, "Estimating the World Cancer Burden: Globocan 2000,” International Journal of Cancer, Vol. 94, No. 2, 2001, pp. 153-156. doi:10.1002/ijc.1440

[6] A. Jemal, et al., "Cancer Statistics, 2008," Cancer Journal for Clinicians, Vol. 58, No. 2, 2008, pp. 71-96. 
doi:10.3322/CA.2007.0010

[7] A. Ohtsu, "Chemotherapy for Metastatic Gastric Cancer: Past, Present, and Future,” Journal of Gastroenterology, Vol. 43, No. 4, 2008, pp. 256-264. doi:10.1007/s00535-008-2177-6

[8] C. S. Fuchs and R. J. Mayer, "Gastric Carcinoma,” The New England Journal of Medicine, Vol. 333, No. 1, 1995, pp. 32-41. doi:10.1056/NEJM199507063330107

[9] Z. Liang, et al., "Analysis of EGFR,HER2, and TOP2A Gene Status and Chromosomal Polysomy in Gastric Adenocarcinoma from Chinese Patients," BMC Cancer, Vol. 8, 2008, p. 363. doi:10.1186/1471-2407-8-363

[10] D. A. Corley and P. A. Buffler, "Oesophageal and Gastric Cardia Adenocarcinomas: Analysis of Regional Variation Using the Cancer Incidence in Five Continents Database," International Journal of Epidemiology, Vol. 30, No. 6, 2001, pp. 1415-1425. doi:10.1093/ije/30.6.1415

[11] T. Takehana, et al., "Status of c-erbB-2 in Gastric Adenocarcinoma: A Comparative Study of Immunohistochemistry, Fluorescence in Situ Hybridization and Enzyme-Linked Immuno-Sorbent Assay,” International Journal of Cancer, Vol. 98, No. 6, 2002, pp. 833-837. doi:10.1002/ijc.10257

[12] L. Albarello, et al., "HER2 Testing in Gastric Cancer," Advances in Anatomic Pathology, Vol. 18, No. 1, 2011, pp. 53-59. doi:10.1097/PAP.0b013e3182026d72

[13] "Cetuximab Approved by FDA for Treatment of Head and Neck Squamous Cell Cancer,” Cancer Biology \& Therapy, Vol. 5, No. 4, 2006, pp. 340-342.

[14] R. M. Giusti, et al., "FDA Drug Approval Summary: Panitumumab (Vectibix),” Oncologist, Vol. 12, No. 5, 2007, pp. 577-583. doi:10.1634/theoncologist.12-5-577

[15] M. H. Cohen, et al., "FDA Drug Approval Summary: Erlotinib (Tarceva) Tablets,” Oncologist, Vol. 10, No. 7, 2005, pp. 461-466. doi:10.1634/theoncologist.10-7-461

[16] P. Laurent-Puig and J. Taieb, "Lessons from Tarceva in Pancreatic Cancer: Where Are We Now, and How Should Future Trials Be Designed in Pancreatic Cancer?” Current Opinion in Oncology, Vol. 20, No. 4, 2008, pp. 454458. doi:10.1097/CCO.0b013e32830218d6

[17] M. V. Karamouzis, et al., "Therapies Directed against Epidermal Growth Factor Receptor in Aerodigestive Carcinomas," Journal of the American Medical Association, Vol. 298, No. 1, 2007, pp. 70-82. doi:10.1001/jama.298.1.70

[18] A. Gonçalves, et al., "A Polymorphism of EGFR Extracellular Domain Is Associated with Progression FreeSurvival in Metastatic Colorectal Cancer Patients Receiving Cetuximab-Based Treatment," BMC Cancer, Vol. 8, 2008, p. 169. doi:10.1186/1471-2407-8-169

[19] P. Lauren, “The Two Histological Main Types of Gastric Carcinoma: Diffuse and so Called Intestinal Type Carcinoma. An Attempt at a Histo-Clinical Classification,” Acta Pathol Microbiol Scand, Vol. 64, 1965, pp. 31-49.

[20] T. Braut, et al., "Epidermal Growth Factor Receptor Protein Expression and Gene Amplification in Normal, Hyperplastic, and Cancerous Glottic Tissue: Immunohistochemical and Fluorescent in Situ Hybridization Study on
Tissue Microarrays,” Croatian Medical Journal, Vol. 50, No. 4, 2009, pp. 370-379. doi:10.3325/cmj.2009.50.370

[21] R. Bhargava, et al., "EGFR Gene Amplification in Breast Cancer: Correlation with Epidermal Growth Factor Receptor mRNA and Protein Expression and HER-2 Status and Absence of EGFR-Activating Mutations," Modern Pathology, Vol. 18, No. 8, 2005, pp. 1027-1033. doi:10.1038/modpathol.3800438

[22] C. G. Kleer, et al., "Pathologic, Immunohistochemical, and Molecular Features of Benign and Malignant Phyllodes Tumors of the Breast,” Modern Pathology, Vol. 14, No. 3, 2008, pp. 185-190. doi:10.1038/modpathol.3880282

[23] T. Dragovich and C. Campen, "Anti-EGFR-Targeted Therapy for Esophageal and Gastric Cancers: An Evolving Concept,” Journal of Oncology, Vol. 2009, 2009, Article ID: 804108. doi:10.1155/2009/804108

[24] E. Mammano, et al., "Epidermal Growth Factor Receptor (EGFR): Mutational and Protein Expression Analysis in Gastric Cancer,” Anticancer Research, Vol. 26, No. 5A, 2006, pp. 3547-3350.

[25] T. Takehana, et al., "Expression of Epidermal Growth Factor Receptor in Gastric Carcinomas,” Clinical Gastroenterology and Hepatology, Vol. 1, No. 6, 2003, pp. 438445. doi:10.1016/S1542-3565(03)00219-2

[26] J. W. Lee, et al., "Absence of EGFR Mutation in the Kinase Domain in Common Human Cancers Besides Non-Small Cell Lung Cancer,” International Journal of Cancer, Vol. 113, No. 3, 2005, pp. 510-511. doi:10.1002/ijc.20591

[27] K. Mimori, et al., “The Epidermal Growth Factor Receptor Gene Sequence Is Highly Conserved in Primary Gastric Cancers,” Journal of Surgical Oncology, Vol. 93, No. 1, 2006, pp. 44-46. doi:10.1002/jso.20426

[28] G. Galizia, et al., "Epidermal Growth Factor Receptor (EGFR) Expression Is Associated with a Worse Prognosis in Gastric Cancer Patients Undergoing Curative Surgery," World Journal of Surgery, Vol. 31, No. 7, 2007, pp. 1458-1468. doi:10.1007/s00268-007-9016-4

[29] J. G. Paez, et al., "EGFR Mutations in Lung Cancer: Correlation with Clinical Response to Gefitinib Therapy," Science, Vol. 304, No. 5676, 2004, pp. 1497-1500. doi:10.1126/science.1099314

[30] H. J. Lee, et al., "Protein Overexpression and Gene Amplification of Epidermal Growth Factor Receptor in Non Small Cell Lung Carcinomas: Comparison of Four Commercially Available Antibodies by Immunohistochemistry and Fluorescence in Situ Hybridization Study," Lung Cancer, Vol. 68, No. 3, 2010, pp. 375-382. doi:10.1016/j.lungcan.2009.07.014

[31] M. Hofmann, et al., "Assessment of a HER2 Scoring System for Gastric Cancer: Results from a Validation Study," Histopathology, Vol. 52, No. 7, 2008, pp. 797805. doi:10.1111/j.1365-2559.2008.03028.x

[32] S. C. Wang and M. C. Hung, "Cytoplasmic/Nuclear Shuttling and Tumor Progression," Annals of the New York Academy of Sciences, Vol. 1059, 2005, pp. 11-15. doi:10.1196/annals.1339.002 
[33] S. Y. Lin, et al., "Nuclear Localization of EGF Receptor and Its Potential New Role as a Transcription Factor," Nature Cell Biology, Vol. 3, No. 9, 2001, pp. 802-808. doi:10.1038/ncb0901-802

[34] H. W. Lo, et al., "Nuclear Interaction of EGFR and STAT3 in the Activation of the iNOS/NO Pathway," Cancer Cell, Vol. 7, No. 6, 2005, pp. 575-589. doi:10.1016/j.ccr.2005.05.007

[35] Lo HW, et al., "EGFR Signaling Pathway in Breast Cancers: From Traditional Signal Transduction to Direct Nuclear Translocalization,” Breast Cancer Research and Treatment, Vol. 95, No. 3, 2006, pp. 211-218. doi:10.1007/s10549-005-9011-0

[36] J. S. de Bono and E. K. Rowinsky, "The ErbB Receptor Family: A Therapeutic Target for Cancer," Trends in Molecular Medicine, Vol. 8, No. 4, 2002, pp. S19-S26. doi:10.1016/S1471-4914(02)02306-7

[37] J. P. Kallio, et al., "Membranous Location of EGFR Immunostaining Is Associated with Good Prognosis in Renal Cell Carcinoma,” British Journal of Cancer, Vol. 89, No. 7, 2003, pp. 1266-1269. doi:10.1038/sj.bjc.6601241

[38] C. Langner, et al., "Are Heterogenous Results of EGFR Immunoreactivity in Renal Cell Carcinoma Related to Non-Standardised Criteria for Staining Evaluation?” Journal of Clinical Pathology, Vol. 57, No. 7, 2004, pp. 773775. doi:10.1136/jcp.2003.015743

[39] G. C. Li, et al., “Are Biomarkers Correlated with Recurrence Patterns in Patients with Resectable Gastric Adenocarcinoma," Molecular Biology Reports, Vol. 39, No. 1, 2012, pp. 399-405. doi:10.1007/s11033-011-0751-0

[40] N. E. Hynes and H. A. Lane, "ERBB Receptors and Cancer: The Complexity of Targeted Inhibitors," Nature Reviews Cancer, Vol. 5, No. 5, 2005, pp. 341-354. doi:10.1038/nrc1609

[41] V. W. Lui, J. R. Grandis, "EGFR-Mediated Cell Cycle
Regulation,” Anticancer Research, Vol. 22, No. 1A, 2002, pp. 1-11.

[42] Tumor Research Center, "The Expression of p53, PCNA, EGFR and Its Relationship to Clinicopathological Features and Prognosis of Gastric Cancer.” http://www.tumorres.com/tumor-biol/37091.htm

[43] Y. Maehara, et al., "Prognostic Value of p53 Protein Expression for Patients with Gastric Cancer-A Multivariate Analysis,” British Journal of Cancer, Vol. 79, No. 7-8, 1999, pp. 1255-1261. doi:10.1038/sj.bjc.6690201

[44] X. P. Liu, et al., "Combined Examination of p27(Kip1), p21(Waf1/Cip1) and p53 Expression Allows Precise Estimation of Prognosis in Patients with Gastric Carcinoma,” Histopathology, Vol. 39, No. 6, 2001, pp. 603610. doi:10.1046/j.1365-2559.2001.01283.x

[45] M. S. Al-Moundhri, et al., "The Prognostic Significance of p53, p27 kip1, p21 waf1, HER-2/Neu, and Ki67 Proteins Expression in Gastric Cancer: A Clinicopathological and Immunohistochemical Study of 121 Arab Patients,” Journal of Surgical Oncology, Vol. 91, No. 4, 2005, pp. 243-252. doi:10.1002/jso.20324

[46] K. Motojima, et al., "Expression of p53 Protein in Gastric Carcinomas Is Not Independently Prognostic,” Surgery, Vol. 116, No. 5, 1994, pp. 890-895.

[47] H. E. Gabbert, et al., "The Relationship of p53 Expression to the Prognosis of 418 Patients with Gastric Carcinoma,” Cancer, Vol. 76, No. 5, 1995, pp. 720-726. doi:10.1002/1097-0142(19950901)76:5<720::AID-CNCR 2820760503>3.0.CO;2-E

[48] V. W. Lui and J. R. Grandis, "EGFR-Mediated Cell Cycle Regulation,” Anticancer Research, Vol. 22, No. 1A, 2002, pp. 1-11.

[49] E. Tahara, "Genetic Pathways of Two Types of Gastric Cancer,” IARC Scientific Publications, Vol. 157, 2004, pp. 327-349. 